\title{
openheart The value of aspartate aminotransferase and alanine aminotransferase in cardiovascular disease risk assessment
}

\author{
Stephen F Weng, ${ }^{1}$ Joe Kai, ${ }^{1}$ Indra Neil Guha, ${ }^{2}$ Nadeem Qureshi ${ }^{1}$
}

To cite: Weng SF, Kai J, Guha IN, et al. The value of aspartate aminotransferase and alanine aminotransferase in cardiovascular disease risk assessment. Open Heart 2015;2:e00272. doi:10.1136/openhrt-2015000272

\section{- Additional material is available. To view please visit the journal (http://dx.doi.org/ 10.1136/openhrt-2015- 000272).}

Received 27 March 2015 Revised 17 July 2015 Accepted 4 August 2015

\section{(1) CrossMark}

${ }^{1}$ Division of Primary Care, NIHR School of Primary Care Research, University of Nottingham, Nottingham, UK ${ }^{2} \mathrm{NIHR}$ Biomedical Research Unit in Gastrointestinal and Liver Diseases at Nottingham University Hospitals NHS Trust and the University of Nottingham, Nottingham, UK

Correspondence to Dr Stephen F Weng; stephen.weng@nottingham. ac.uk

\section{ABSTRACT}

Objective: Aspartate aminotransferase to alanine aminotransferase (AST/ALT) ratio, reflecting liver disease severity, has been associated with increased risk of cardiovascular disease (CVD). The aim of this study was to evaluate whether the AST/ALT ratio improves established risk prediction tools in a primary care population.

Methods: Data were analysed from a prospective cohort of 29316 UK primary care patients, aged 25-84 years with no history of CVD at baseline. Cox proportional hazards regression was used to derive 10-year multivariate risk models for the first occurrence of CVD based on two established risk prediction tools (Framingham and QRISK2), with and without including the AST/ALT ratio. Overall, model performance was assessed by discriminatory accuracy (AUC c-statistic).

Results: During a total follow-up of 120462 personyears, 782 patients (59\% men) experienced their first CVD event. Multivariate models showed that elevated AST/ALT ratios were significantly associated with CVD in men (Framingham: HR 1.37, 95\% $\mathrm{Cl} 1.05$ to 1.79 ; QRISK2: HR $1.40,95 \% \mathrm{Cl} 1.04$ to 1.89 ) but not in women (Framingham: HR 1.06, 95\% $\mathrm{Cl} 0.78$ to 1.43 ; QRISK2: HR $0.97,95 \% \mathrm{Cl} 0.70$ to 1.35 ). Including the AST/ALT ratio with all Framingham risk factors (AUC c-statistic: $0.72,95 \% \mathrm{Cl} 0.71$ to 0.74 ) or QRISK2 risk factors (AUC c-statistic: $0.73,95 \% \mathrm{Cl} 0.71$ to 0.74 ) resulted in no change in discrimination from the established risk prediction tools. Limiting analysis to those individuals with raised ALT showed that discrimination could improve by $5 \%$ and $4 \%$ with Framingham and QRISK2 risk factors, respectively. Conclusions: Elevated AST/ALT ratio is significantly associated with increased risk of developing CVD in men but not women. However, the ratio does not confer any additional benefits over established CVD risk prediction tools in the general population, but may have clinical utility in certain subgroups.

\section{INTRODUCTION}

Cardiovascular disease (CVD) continues to be the leading cause of death in the developed world. In the UK, there are an estimated 180000 deaths per year from CVD. ${ }^{1}$ The UK National Institute for Health and

\section{KEY MESSAGES}

What is already known about this subject?

- The aspartate aminotransferase to alanine aminotransferase (AST/ALT) ratio, reflecting liver disease severity, has been associated with increased risk of cardiovascular disease (CVD) in patients with liver disease. It is unknown whether these simple, routinely collected biomarkers, can improve CVD prediction in a general primary care population.

What does this study add?

- By following-up a large UK cohort of primary care patients over 10 years, we have found that the AST/ALT ratio is significantly associated with increased risk of CVD in men but not women. However, the ratio does not confer any additional benefits in predictive accuracy for predicting CVD when included in standard primary care-based risk prediction tools.

How might this impact on clinical practice?

- Our analysis suggests the AST/ALT ratio should not be included in current CVD risk prediction tools for the general primary care population. However, in the era of stratified medicine, those with raised AST/ALT ratio may represent a higher risk subgroup that could benefit from closer monitoring, particularly when the ALT is raised.

Care Excellence (NICE) lipid modification guidelines recommend the use of risk prediction tools, to identify individuals at increased risk of CVD. ${ }^{2}$ In the UK, QRISK2 ${ }^{3}$ is recommended while internationally, the Framingham tool $^{4}$ is adopted. These tools calculate CVD risk using recognised risk factors, such as hypertension, cholesterol levels, age, smoking and family history of premature CVD.

Although most CVD events can be attributed to the aforementioned major risk factors embedded within routinely-used risk prediction tools, a significant proportion of the population will experience an event in the absence of traditional risk factors. For 
example, up to $50 \%$ of all myocardial infarctions (MI) and strokes occur in individuals with low-density lipoprotein cholesterol levels below the recommended thresholds for lipid modification. ${ }^{5}$ In the USA, the National Cholesterol Education Program (NCEP) Adult Treatment Panel (ATP) III now recommends that to effectively reduce the CVD risk of such patients requires consideration of 'emerging risk factors' ${ }^{6}$

Liver function enzymes aspartate aminotransferase (AST) and alanine aminotransferase (ALT) are examples of emerging biomarkers for CVD risk. The AST to ALT has been shown to reflect disease severity in a number of chronic liver diseases, including alcoholic and non-alcoholic liver disease, ${ }^{7}$ autoimmune liver disease $^{8}$ and hepatitis C. ${ }^{9}$ Previous studies ${ }^{10-14}$ have demonstrated that CVD is the leading cause of death in non-alcoholic fatty liver disease (NAFLD), with higher rates coinciding with higher liver-related mortality over follow-up periods from 10 to 20 years.

The detection and identification of liver disease in the community is limited. Liver disease is asymptomatic until the final stages of cirrhosis. However, using the enzymes in a simple ratio (AST/ALT) or as a component of panel marker tests has been shown to have diagnostic accuracy for significant liver disease. ${ }^{15}{ }^{16} \mathrm{In}$ addition, these surrogates of liver injury improve the prediction of future clinical events including cardiovascular outcomes in patients with liver disease. ${ }^{17} 18$ However, it is still unknown whether the AST/ALT ratio can improve prediction for cardiovascular outcomes in a general primary care population. These simple liver markers are routinely available in primary care, offering potential utility in primary care-based risk prediction models. Therefore, the aim of this study was to evaluate whether the inclusion of AST/ALT ratio improves standard 10-year risk prediction models for CVD in a primary care population.

\section{METHODS}

\section{Data sources}

Data were obtained from the Clinical Practice Research Datalink (CPRD), a cohort of patients with prospectively collected data, derived from anonymised electronic medical records of more than 12 million patients from 681 UK general practices. Approximately $8 \%$ of the UK population is included and the database is broadly representative of the UK population. ${ }^{19}$ Additionally, $55 \%$ of the CPRD patients are also linked to secondary care across UK, through Hospital Episode Statistics (HES) database, which document hospital admissions, diagnoses (ICD-10), and treatment received.

Data undergo quality checks and practices are designated as meeting internal quality criteria for research purposes and over 550 peer-reviewed studies have been published from the databases. ${ }^{19}$ Ethical approval was granted by the CPRD Independent Scientific Advisory Committee (protocol 14_080).

\section{Study population}

The study population consisted of registered patients at a CPRD General Practice aged between 25 and 84 years (covering the age range of the CVD risk prediction tool) at baseline, with complete AST and ALT measurements recorded within 2 days of one another prior to the baseline date, and who had complete data recorded on seven recognised core risk factors (sex, age, smoking status, systolic blood pressure, blood pressure treatment, total cholesterol (TC) and high-density lipoprotein (HDL) cholesterol) prior to the baseline date. The baseline date was specified as 1 January 2004, which enabled patients to have a 10-year follow-up for estimating 10 -year CVD risk. The end of the study period was the most recent date for which data were available (1 January 2014). Patients were excluded if they had a pre-existing history of CVD or inherited lipid disorders prior to baseline, were on lipid lowering drugs prior to baseline, or outside the specified age range at baseline.

\section{Risk factors}

The core risk factors for predicting CVD were derived from the variables included in the 10-year Framingham and QRISK2 risk algorithms (table 1). The core factors encompass all eight variables in the Framingham risk algorithm (age, gender, systolic blood pressure, blood pressure treatment, TC, HDL cholesterol, diabetes and smoking). The QRISK2 risk algorithms consists of 14 variables, including all risk factors present in the Framingham, with the addition of body mass index (BMI), family history of premature coronary heart disease (CHD), chronic kidney disease, atrial fibrillation, rheumatoid arthritis, Townsend deprivation score and ethnicity. Blood pressure treatment was classified as a diagnosis of hypertension and at least one current prescription of an antihypertensive agent. The AST/ALT ratio was included as a novel marker to these original risk algorithms. We used these risk factors to derive new sets of regression functions which were calibrated for the CPRD study population, a common approach in risk prediction modelling. ${ }^{20}$

\section{Outcome}

The outcome of interest for this analysis was a diagnosis of the first CVD event (fatal or non-fatal) in a patient's electronic medical records during the follow-up period of 10 years after the baseline date (1 January 2014). The medical diagnosis of CVD is coded electronically in National Health Service (NHS) primary care electronic health records as Read Codes (full details in online supplementary materials). Further, for those patients who were linked to secondary care (HES) during the specified study period (55\% of sample), we utilised ICD-10 codes (I20-25 for coronary heart disease, I60-69 for cerebrovascular disease) to validate the CVD outcome recorded in primary care. If the secondary care CVD event recorded occurred prior to the primary care CVD event, then the date of the first event was determined by 
Table 1 Risk factors included in the standard 10-year Framingham and QRISK2 cardiovascular risk prediction algorithms

\begin{tabular}{|c|c|c|}
\hline Risk factors & Framingham risk factors & QRISK2 risk factors \\
\hline Age (years) & $\checkmark$ & $\checkmark$ \\
\hline Gender (male/female) & $\checkmark$ & $\checkmark$ \\
\hline Systolic blood pressure (mm Hg) & $\checkmark$ & $\checkmark$ \\
\hline Blood pressure treatment (yes/no) & $\checkmark$ & \\
\hline Total cholesterol (mmol/L) & $\checkmark$ & \\
\hline HDL cholesterol (mmol/L) & $\checkmark$ & $\checkmark$ \\
\hline Total cholesterol/HDL ratio & & $\checkmark$ \\
\hline Body mass index $\left(\mathrm{kg} / \mathrm{m}^{2}\right)$ & & $\checkmark$ \\
\hline Diabetes (yes/no) & $\checkmark$ & $\checkmark$ \\
\hline Family history of $\mathrm{CHD}<60$ years (yes $/ \mathrm{no}$ ) & & $\checkmark$ \\
\hline Chronic kidney disease (yes/no) & & $\checkmark$ \\
\hline Atrial fibrillation (yes $/ \mathrm{no}$ ) & & $\checkmark$ \\
\hline Rheumatoid arthritis (yes/no) & & $\checkmark$ \\
\hline Smoking (yes/no) & $\checkmark$ & $\checkmark$ \\
\hline Ethnicity (Caucasian/non-Caucasian) & & $\checkmark$ \\
\hline Townsend score (quintiles) & & $\checkmark$ \\
\hline AST/ALT ratio* & & \\
\hline
\end{tabular}

the secondary care record and vice versa. Patients who died due to other causes or transferred before the end of follow-up were censored from the analysis.

\section{Statistical analysis}

As the Framingham risk scores could be calculated from the core risk factors, there were no missing values. However, to calculate QRISK2 risk scores, there were missing values for additional variables of BMI, ethnicity and Townsend deprivation score. Standard procedure of multiple imputation was used to deal when missing values where identified in QRISK2 variables with 10 copies of the missing data imputed. This approach follows the same imputation procedures utilised in the original QRISK2 algorithm. ${ }^{3}$ A descriptive analysis was performed, reporting number (\%), mean (SD) and median (IQR) for categorical, normal continuous and non-normal continuous variables, respectively.

Multivariate prediction models were derived from Cox proportional hazards regression, using the original risk factors included in Framingham and QRISK2, which gave the probability of the first onset of the CVD outcome. All continuous variables were log-transformed to preserve normality in the multivariate models. In addition to patients being censored as a result of loss to follow-up (death or transfer), patients who were prescribed lipid-lowering drug treatment, during follow-up, were also censored as these patients would likely have significantly altered their risk during the follow-up period. We then incorporated the AST/ALT ratio into both multivariate models to derive a new set of prediction models. These functions were then applied to each patient to calculate a new patient risk score with the addition of the AST/ALT ratio. The process of developing the linear prediction model from Cox regression to calculate patient 10-year CVD risk is described in detail in online supplementary materials. All underlying assumptions were investigated for the Cox models including proportional hazards, influential and outlying observations.

The performance of the multivariate prediction models was assessed by the area under the receiver operating characteristics curves (AUC), estimated as Harrell's c-statistic, with higher values representing better discrimination (ability to distinguish between a case and a non-case). To generate CIs for the c-statistic, a jack-knife procedure $^{21}$ was used to bootstrap SEs. All analyses were performed using STATAV.13 MP4.

\section{RESULTS}

\section{Characteristics of the study population}

There were 30929 patients at baseline (1 January 2004) with a documented AST/ALT ratio and complete data for eight core risk factors (age, gender, systolic blood pressure, blood pressure treatment, TC, HDL cholesterol, diabetes and smoking). From this starting sample, 1613 patients were removed due to outlying observations for cholesterol and blood pressure, or data entry errors (non-numerical entries). The outlying observations were determined by reference ranges, plus/or minus $10 \%$, determined from data extracted from the Health Survey for England ${ }^{22}$ (ranges shown in table 2). This process is a validated approach ${ }^{23}$ in removing outliers for UK general practice database studies using the Health Survey for England.

The remaining 29316 patients $(52 \%$ female $)$ were included for the analysis as the study cohort in which patients were followed-up for a total of 120462 personyears (men: 55606 person-years; women: 64856 person- 
Table 2 Reference ranges derived from data extracted from the 2012 health survey for England

\begin{tabular}{lll}
\hline \multirow{2}{*}{ Variable } & \multicolumn{2}{l}{ Reference ranges } \\
\cline { 2 - 3 } & Men & Women \\
\hline Total cholesterol $(\mathrm{mmol} / \mathrm{L})$ & $2.1-10.0$ & $2.3-11.9$ \\
HDL cholesterol $(\mathrm{mmol} / \mathrm{L})$ & $0.4-3.7$ & $0.5-5.0$ \\
Systolic blood pressure $(\mathrm{mm} \mathrm{Hg})$ & $70-220$ & $59-221$ \\
Body mass index $\left(\mathrm{kg} / \mathrm{m}^{2}\right)$ & $7.5-65.3$ & $10.3-61.8$ \\
\hline HDL, high-density lipoprotein. & &
\end{tabular}

years). During the follow-up period, there were 1241 patients $(4.2 \%)$ who died due to other causes and 2701 $(9.2 \%)$ patients who were lost to follow-up due to transferring from the practice. Additionally, 2085 patients $(7.1 \%)$ were censored due to starting statin treatment during the follow-up period. The remaining patients were alive and at the practice at the end of follow-up. There were a total of 782 patients (461 men, 321 women) who had a CVD event, with a slightly higher proportion occurring in men than in women. The median age of the cohort for men was 58 years (IQR:
49-68) and for women was 61 years (IQR: 52-71). The median AST/ALT differed between men and women (0.8 IQR: 0.7-1.1 for men; 1.0 IQR: 0.8-1.3 for women). Median TC and HDL cholesterol were lower for men than in women while median systolic blood pressure and BMI were similar between men and women. Missing data also occurred in three variables used in the QRISK2 algorithm. BMI was not recorded in $72 \%$ of men and $73 \%$ of women, ethnicity missing for $51 \%$ of men and $48 \%$ of women, and Townsend score was missing for $36 \%$ of men and $38 \%$ of women. Further details of all descriptive characteristics are presented in table 3 .

\section{Multivariate hazard models}

Risk factors in Framingham risk prediction model

Two multivariate hazard regression models were developed for both the Framingham and QRISK2 risk factors. The Framingham risk factor model shown in table 4 includes all original Framingham risk factors with the addition of the AST/ALT ratio.

Table 3 Characteristics of patients aged 25-84 years in the CPRD study cohort stratified by gender with a 10-year follow-up

\begin{tabular}{|c|c|c|}
\hline Characteristics & $\mathbf{N}(\%)$ of men & $\mathbf{N}(\%)$ of women \\
\hline Patients & $14175(0.48)$ & $15141(0.52)$ \\
\hline Total person-years & 55606 & 64856 \\
\hline CVD event & $461(0.03)$ & $321(0.02)$ \\
\hline Median age (IQR) & $58(49-68)$ & $61(52-71)$ \\
\hline Median total cholesterol in mmol/L (IQR) & $5.3(4.6-6.0)$ & $5.5(4.8-6.3)$ \\
\hline Median HDL cholesterol in mmol/L (IQR) & $1.3(1.1-1.5)$ & $1.5(1.3-1.8)$ \\
\hline Median total cholesterol/HDL ratio (IQR) & $4.2(3.4-5.0)$ & $3.6(2.9-4.4)$ \\
\hline Median systolic blood pressure in $\mathrm{mmol} / \mathrm{L}$ (IQR) & $140(128-150)$ & $140(126-150)$ \\
\hline Median body mass index in $\mathrm{kg} / \mathrm{m}^{2}(\mathrm{IQR})^{*}$ & $26.3(23.3-30.0)$ & $26.4(22.5-31.1)$ \\
\hline Body mass index missing & $12554(0.89)$ & $13339(0.88)$ \\
\hline Median AST/ALT ratio (IQR) & $0.8(0.7-1.1)$ & $1.0(0.8-1.3)$ \\
\hline On blood pressure treatment & $3070(0.22)$ & $3559(0.24)$ \\
\hline Smoking & $3222(0.23)$ & $3066(0.20)$ \\
\hline Diabetes & $2678(0.19)$ & $2305(0.15)$ \\
\hline Chronic kidney disease & $124(0.009)$ & $84(0.006)$ \\
\hline Atrial fibrillation & $386(0.03)$ & $315(0.02)$ \\
\hline Rheumatoid arthritis & $118(0.008)$ & $296(0.02)$ \\
\hline Family history of premature $\mathrm{CHD}<60$ years & $719(0.05)$ & $771(0.05)$ \\
\hline \multicolumn{3}{|l|}{ Ethnicity* } \\
\hline Caucasian & $5522(0.39)$ & $6493(0.43)$ \\
\hline Non-Caucasian & $1368(0.10)$ & $1382(0.09)$ \\
\hline Missing & $7285(0.51)$ & $7266(0.48)$ \\
\hline \multicolumn{3}{|l|}{ Townsend quintiles } \\
\hline 1st-least deprived & $2739(0.19)$ & $2499(0.17)$ \\
\hline 2nd & $2085(0.15)$ & $2046(0.14)$ \\
\hline $3 r d$ & $1679(0.12)$ & $1875(0.12)$ \\
\hline 4th & $1622(0.11)$ & $1837(0.12)$ \\
\hline 5th-most deprived & $940(0.06)$ & $1149(0.08)$ \\
\hline Missing & $5120(0.36)$ & $5735(0.38)$ \\
\hline
\end{tabular}

*Variable contains missing values.

Values are number and proportions unless otherwise stated.

ALT, alanine transaminase; AST, aspartate transaminase; CVD, cardiovascular disease; CHD, coronary heart disease; CRPD, Clinical Practice Research Datalink; HDL, high-density lipoprotein. 
Table 4 Multivariate hazard model showing adjusted HRs and corresponding $95 \% \mathrm{Cl}$ for calibrated Framingham risk factors including AST/ALT ratio

\begin{tabular}{|c|c|c|}
\hline \multirow[b]{2}{*}{ Risk factors } & \multicolumn{2}{|l|}{ Adjusted HR (95\% Cl) } \\
\hline & Men & Women \\
\hline Age (years) ${ }^{*}$ & $53.33(30.64$ to 92.83$) \dagger$ & 158.65 (76.38 to 329.55$) \dagger$ \\
\hline Total cholesterol $(\mathrm{mmol} / \mathrm{L})^{*}$ & 1.05 (0.63 to 1.77$)$ & $1.10(0.58$ to 2.09$)$ \\
\hline HDL cholesterol (mmol/L)* & $0.53(0.37$ to 0.76$) \dagger$ & $0.43(0.28$ to 0.66$) \dagger$ \\
\hline Systolic blood pressure if treated $(\mathrm{mm} \mathrm{Hg})^{\star}$ & $2.07(1.02$ to 4.43$) \dagger$ & $2.62(1.06$ to 6.52$) \dagger$ \\
\hline Systolic blood pressure if no treatment $(\mathrm{mm} \mathrm{Hg})^{*}$ & $2.05(1.01$ to 4.36$) \dagger$ & $2.67(1.08$ to 6.58$) \dagger$ \\
\hline \multicolumn{3}{|l|}{ Smoking } \\
\hline No & Ref & Ref \\
\hline Yes & $1.48(1.20$ to 1.83$) \dagger$ & $2.09(1.61$ to 2.70$) \dagger$ \\
\hline \multicolumn{3}{|l|}{ Diabetes } \\
\hline No & Ref & Ref \\
\hline Yes & 1.35 (1.02 to 1.77$) \dagger$ & $1.69(1.16$ to 2.46$) \dagger$ \\
\hline AST/ALT ratio* & $1.37(1.05$ to 1.79$) \dagger$ & $1.06(0.78$ to 1.43$)$ \\
\hline
\end{tabular}

Age was the most dominant predictor of CVD for men and women. Systolic blood pressure, smoking, diabetes significantly increased risk of CVD while higher HDL cholesterol significantly reduced risk of CVD for men and women. TC levels were not found to be significantly associated with CVD. Higher AST/ALT ratio significantly increased risk of CVD in men but not women.

\section{Risk factors in QRISK2 risk prediction model}

The QRISK2 risk factor model shown in table 5 includes all original QRISK2 risk factors with the addition of the AST/ALT ratio.

Similar to the Framingham risks factors model, the effect sizes of the core risk factors in the QRISK2 was similar. Again, age was the most dominant predictor of CVD for men and women. Systolic blood pressure, TC to HDL cholesterol ratio, smoking and diabetes were significantly associated with increased risk of CVD for men and women. Additional risk factors of chronic kidney disease, and atrial fibrillation were also associated with an increased risk of CVD for men and women. Townsend index showed that living in more deprived areas generally increased risk of CVD but this relationship was not significant in women. However, BMI, blood pressure treatment, rheumatoid arthritis and ethnicity were not found to be associated with CVD. The AST/ ALT ratio was significantly associated with an increase in risk of CVD in men but not women.

\section{Discrimination analysis}

Receiver operating curves were derived for several models in table 6 .

The first set of models excludes the AST/ALT ratio. A simple age and gender-adjusted model (Model 1a) resulted in an AUC c-statistic of 0.68 (95\% CI 0.66 to $0.70)$. The addition of all Framingham risk factors (Model 2a) improved discrimination to 0.72 (95\% CI
0.70 to 0.74 ) while the addition of QRISK2 risk factors (Model 3a) further improved discrimination to 0.73 (95\% CI 0.71 to 0.74 ). Including the AST/ALT ratio in a simple age and gender-adjusted model (Model 1b) slightly improved discrimination to 0.69 (95\% CI 0.67 to 0.71 ) from the age and gender adjusted model (Model 1a), although this increase was not significant. Including the AST/ALT ratio with Framingham risk factors (AUC c-statistic: $0.72,95 \%$ CI 0.71 to 0.74 , Model 2b) or QRISK2 risk factors (AUC c-statistic: $0.73,95 \%$ CI 0.71 to 0.74 , Model $3 \mathrm{~b}$ ) resulted in no change in discrimination from the standard Framingham or QRISK2 risk factor models (Models 2a and 3a). Figure 1 shows no incremental change in discrimination for Framingham and QRISK2 risk factors models with the addition of the AST/ALT ratio. The age and gender model serves as a baseline model for comparison.

Two models were developed to assess the utility of the AST/ALT ratio compared against the standard biomarkers (systolic blood pressure and TC/HDL ratio). The comparator models of age and gender with either systolic blood pressure or the TC to HDL cholesterol ratio resulted in the same discrimination (AUC c-statistic: $0.69,95 \%$ CI 0.67 to 0.71 ) as Model $1 \mathrm{~b}$ including the AST/ALT ratio. Figure 2 shows that the discrimination increased by $1 \%$ with the inclusion of the AST/ALT ratio with age and gender, resulting in the same incremental benefit in discrimination as including systolic blood pressure or the TC/HDL ratio with age and gender.

\section{Sensitivity analysis}

To take account of previous studies demonstrating the predictive value of the AST/ALT ratio for CVD, as previously reported in patients with NAFLD, is almost always associated with elevated ALT, a further sensitivity analysis was performed. To investigate the effects of this, we 
Table 5 Multivariate hazard model showing adjusted HRs and $95 \% \mathrm{Cl}$ for calibrated QRISK2 risk factors including AST/ALT ratio

\begin{tabular}{|c|c|c|}
\hline \multirow[b]{2}{*}{ Risk factors } & \multicolumn{2}{|l|}{ Adjusted HR (95\% Cl) } \\
\hline & Men & Women \\
\hline Age (years) $)^{*}$ & $63.80(34.65-117.48) \dagger$ & $112.76(50.92-249.70) \dagger$ \\
\hline Total cholesterol/HDL cholesterol ratio* & $1.33(1.01-1.92) \dagger$ & $2.05(1.33-3.16) \dagger$ \\
\hline Systolic blood pressure $(\mathrm{mm} \mathrm{Hg})^{*}$ & $1.84(1.04-3.96) \dagger$ & $2.80(1.12-7.02) \dagger$ \\
\hline Body mass index $\left(\mathrm{kg} / \mathrm{m}^{2}\right)^{\star}$ & $2.84(0.84-9.60)$ & $0.62(0.16-2.33)$ \\
\hline \multicolumn{3}{|l|}{ Blood pressure treatment } \\
\hline No & Ref & Ref \\
\hline Yes & $0.89(0.71-1.12)$ & $1.08(0.83-1.39)$ \\
\hline \multicolumn{3}{|l|}{ Smoking } \\
\hline No & Ref & Ref \\
\hline Yes & $1.51(1.21-1.89) \dagger$ & $1.97(1.50-2.59) \dagger$ \\
\hline \multicolumn{3}{|l|}{ Diabetes } \\
\hline No & Ref & Ref \\
\hline Yes & $1.36(1.03-1.79) \dagger$ & $1.84(1.27-2.66) \dagger$ \\
\hline \multicolumn{3}{|l|}{ Chronic kidney disease } \\
\hline No & Ref & Ref \\
\hline Yes & $1.97(1.02-3.99) \dagger$ & $2.85(1.05-7.74) \dagger$ \\
\hline \multicolumn{3}{|l|}{ Atrial fibrillation } \\
\hline No & Ref & Ref \\
\hline Yes & $1.91(1.32-2.76) \dagger$ & $2.32(1.49-3.62) \dagger$ \\
\hline \multicolumn{3}{|l|}{ Rheumatoid arthritis } \\
\hline No & Ref & Ref \\
\hline Yes & $1.50(0.74-3.02)$ & $1.47(0.80-2.69)$ \\
\hline \multicolumn{3}{|l|}{ Townsend fifths } \\
\hline 1st-least deprived & Ref & Ref \\
\hline 2nd & $1.12(0.86-1.46)$ & $0.96(0.69-1.32)$ \\
\hline $3 r d$ & $1.37(1.04-1.79) \dagger$ & $0.86(0.61-1.21)$ \\
\hline 4th & $1.33(1.02-1.74) \dagger$ & $1.16(0.84-1.60)$ \\
\hline 5th—most deprived & $1.12(0.77-1.62)$ & $1.28(0.88-1.86)$ \\
\hline \multicolumn{3}{|l|}{ Ethnicity } \\
\hline Caucasian & Ref & Ref \\
\hline Non-Caucasian & $0.75(0.56-0.99)$ & $0.86(0.61-1.21)$ \\
\hline AST/ALT Ratio* & $1.40(1.04-1.89) \dagger$ & $0.97(0.70-1.35)$ \\
\hline
\end{tabular}

\begin{tabular}{|c|c|c|c|}
\hline Models & AUC c-statistic & $\mathrm{SE}^{\star}$ & $95 \% \mathrm{Cl}$ \\
\hline \multicolumn{4}{|l|}{ AST/ALT excluded } \\
\hline Model 1a: Age+gender & 0.68 & 0.009 & 0.66 to 0.70 \\
\hline Model 2a: Framingham risk factors & 0.72 & 0.009 & 0.70 to 0.74 \\
\hline Model 3a: QRISK2 risk factors & 0.73 & 0.008 & 0.71 to 0.74 \\
\hline \multicolumn{4}{|l|}{ AST/ALT ratio included } \\
\hline Model 1b: Age+gender+AST/ALT ratio & 0.69 & 0.009 & 0.67 to 0.71 \\
\hline Model $2 \mathrm{~b}$ : Framingham risk factors+AST/ALT ratio & 0.72 & 0.009 & 0.71 to 0.74 \\
\hline Model 3b: QRISK2 risk factors+AST/ALT ratio & 0.73 & 0.008 & 0.71 to 0.74 \\
\hline \multicolumn{4}{|l|}{ Comparator models } \\
\hline Model 4: Age+gender+systolic blood pressure & 0.69 & 0.009 & 0.67 to 0.71 \\
\hline Model 5: Age+gender+TC/HDL ratio & 0.69 & 0.009 & 0.67 to 0.71 \\
\hline
\end{tabular}

Higher area under receiver operating curve (AUC c-statistic) shows better model performance.

SE estimated by jack-knife procedure.

ALT, alanine transaminase; AST, aspartate transaminase; HDL, high-density lipoprotein; TC, total cholesterol. 

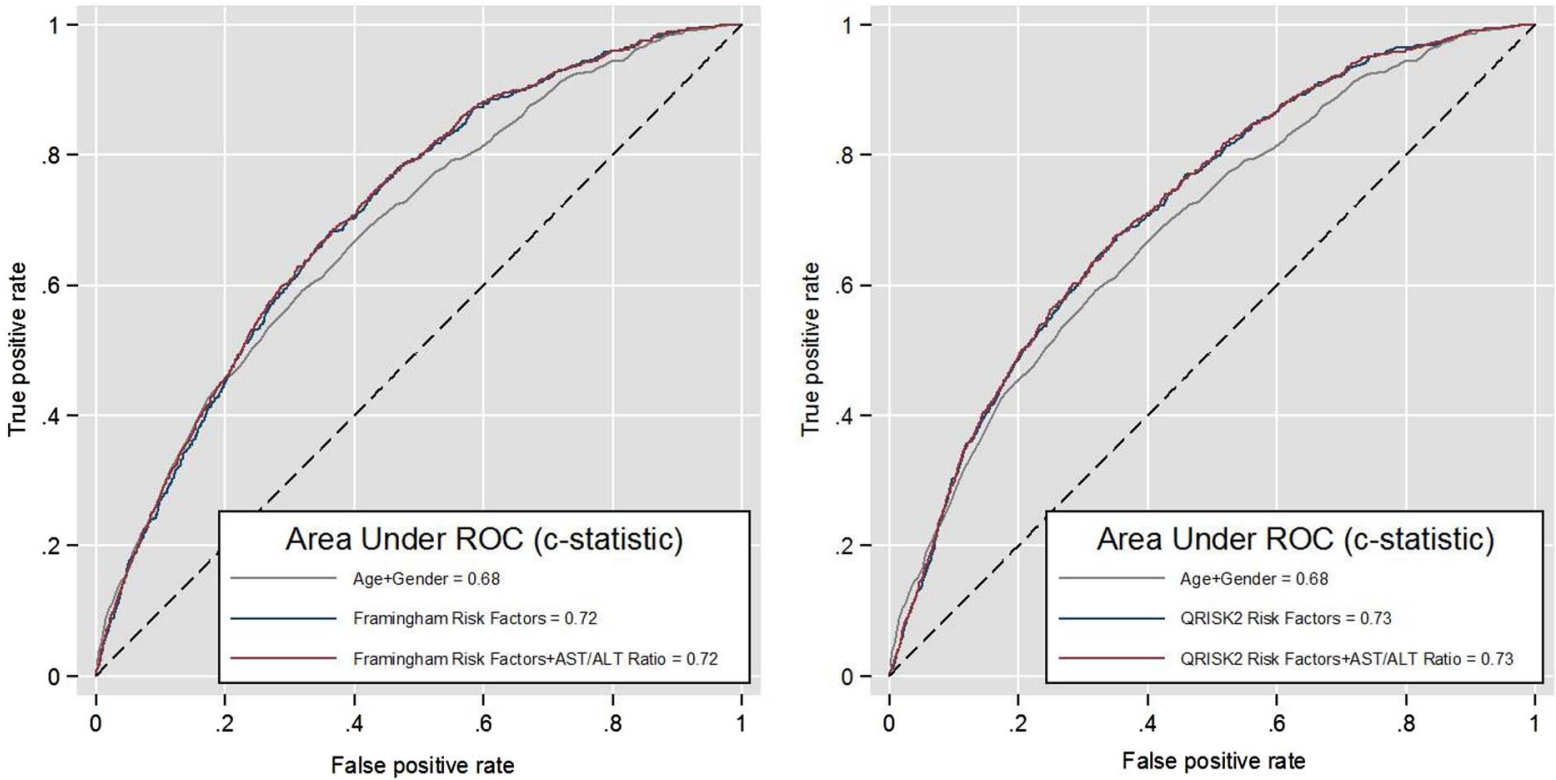

Figure 1 Receiver operating curves (ROC) of the Framingham and QRISK2 risk factor models for predicting 10-year risk of cardiovascular disease with and without the inclusion of the AST/ALT ratio. ALT, alanine transaminase; AST, aspartate transaminase.

excluded patients with normal of low ALT using updated thresholds $<19 \mathrm{U} / \mathrm{L}$ in women and $<30 \mathrm{U} / \mathrm{L}$ in men. ${ }^{24}$ Discrimination of Framingham and QRISK2 CVD prediction models excluding these patients is shown in table 7. Compared to the primary analysis including all patients, this subgroup showed large improvements in

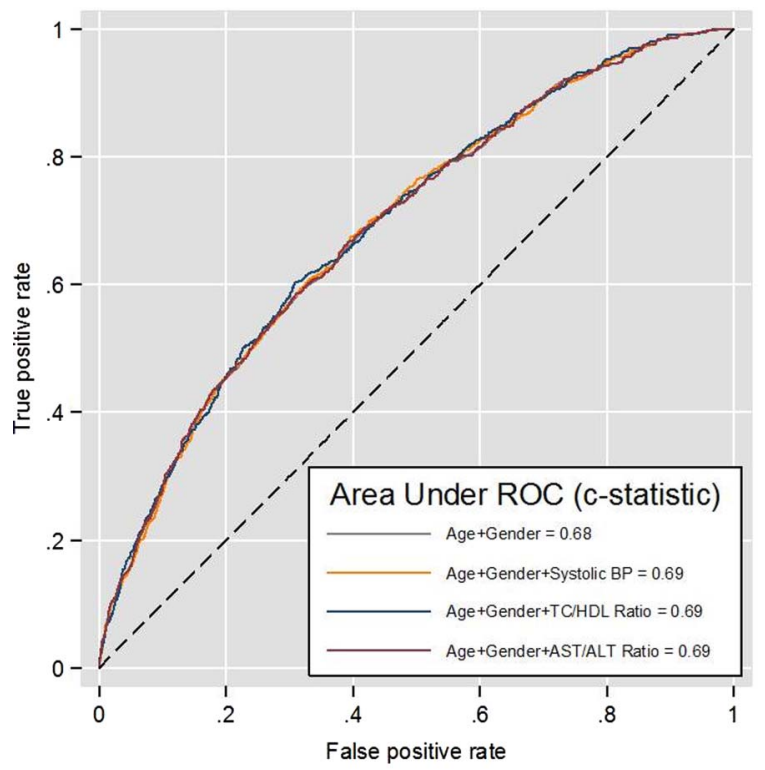

Figure 2 Receiver operating curves (ROC) of simple age and gender adjusted risk factor models for predicting 10-year risk of cardiovascular disease comparing the incremental benefits in discrimination from the AST/ALT ratio, systolic blood pressure, and the TC/HDL ratio. ALT, alanine transaminase; AST, aspartate transaminase; BP, blood pressure; HDL, high-density lipoprotein; TC, total cholesterol. predicting CVD when the AST/ALT ratio was included, resulting in a 5\% and $4 \%$ increase in discrimination using the Framingham and QRISK2 risk factor models, respectively.

\section{DISCUSSION}

There was no improvement in discrimination after the AST/ALT ratio was included in multivariate risk prediction models for CVD in a heterogeneous primary care population. Despite this, we have established in this study that elevated levels of the AST/ALT ratio are independently associated with increased risk of developing CVD within 10 years in men but not women. Additionally, by excluding patients with normal or low ALT levels, we have shown that the AST/ALT ratio confers larger benefits in predicting CVD in individuals with elevated ALT levels. Currently, there is no clear biological mechanism but as well as a marker of CVD risk, AST/ALT ratio stratifies severity of liver disease, and this is independent of features of the metabolic syndrome. ${ }^{25}$ Moreover, emerging evidence in NAFLD suggests that liver fibrosis (with AST/ALT ratio as a proxy marker) stratifies future CVD risk rather than steatosis alone. $^{12} 1418$

The AST/ALT ratio being associated with CVD in men may be related to higher prevalence of liver disease in men. For instance, nearly $60 \%$ of incident cases of cirrhosis in the UK from 1998 to 2009 diagnosed in primary care occurred in men. ${ }^{26}$ Further, the risk of NAFLD increases with being obese or insulin resistant, which again are more common in men. As diabetes is an established risk factor for CVD in all established risk prediction tools while also being a primary risk factor of 
Table 7 Discrimination of the prediction models derived from multivariate hazard models excluding patients with normal or low ALT

\begin{tabular}{llll}
\hline Models & AUC c-statistic & SE $^{*}$ & $\mathbf{9 5 \%} \mathbf{C l}$ \\
\hline AST/ALT excluded & & & \\
$\quad$ Framingham risk factors & 0.66 & 0.013 & 0.64 to 0.69 \\
QRISK2 risk factors & 0.68 & 0.014 & 0.65 to 0.71 \\
AST/ALT ratio included & & & \\
$\quad$ Framingham risk factors+AST/ALT ratio & 0.71 & 0.013 & 0.68 to 0.73 \\
QRISK2 Risk factors+AST/ALT ratio & 0.72 & 0.013 & 0.69 to 0.74 \\
\hline
\end{tabular}

Higher area under receiver operating curve (AUC c-statistic) shows better model performance.

*SE estimated by jack-knife procedure.

$\mathrm{ALT}$, alanine transaminase; AST, aspartate transaminase.

NAFLD, ${ }^{27}$ the effects of elevated AST/ALT ratio is likely masked by diabetes being present in the model. This was likely the case in this study as elevated AST/ALT ratios were also strongly and significantly associated with diabetes $(\mathrm{OR}=2.51,95 \%$ CI 2.31 to 2.72). However, when we removed diabetes as well as other established risk factors, a simple age and gender adjusted model including AST/ALT ratio had a similar incremental improvement in predictive accuracy than other wellestablished GVD risk factors, such as, TC/HDL and systolic blood pressure. This implies that the AST/ALT ratio may have some clinical utility in CVD risk prediction, which is lost when diabetes is incorporated into the model.

\section{CLINICAL IMPLICATIONS}

Our analysis suggests that the AST/ALT ratio should not be included in current CVD risk prediction tools for the general primary care population. However, in the era of stratified medicine, those with raised AST/ALT ratio may represent a higher risk subgroup that could benefit from closer monitoring, particularly when ALT is raised. Latest UK guidelines ${ }^{2}{ }^{28}$ in primary care recommend clinicians to exclude type I diabetic patients in the use of CVD risk assessment tools as the calculated CVD risk may not be reliable, particularly in younger patients. As diabetes is a spectrum of disease which has been simplified as a binary variable in all standard CVD risk tools, a continuous variable such as the AST/ALT ratio may confer additional advantages for further stratification of these higher risk subgroups. Other potential risk factors for liver disease in subgroup populations with high obesity and alcohol usage should also warrant further analysis in which the AST/ALT ratio may confer larger benefits. Emerging evidence (including the findings in this study) showing the utility the AST/ALT ratio in stratifying liver disease and CVD risk in certain subgroups may inform future guideline development as stronger research evidence emerges. Given that the cost of either analyte is relatively cheap, the AST/ALT ratio's utility in predicting future CVD risk in groups such as those with elevated ALT, type II diabetes, and features of metabolic syndrome, alcohol usage or a combination of these factors may be extremely cost-effective. More broadly, the US Preventive Services Task Force, ${ }^{29}$ American Heart Association/American College of Cardiology $^{30}$ and the recent Joint British Societies ${ }^{28}$ recommend now recommend revisiting the value of novel markers in risk prediction tools as more evidence becomes available.

In the context of identifying patients who should not be prescribed statin, the latest NICE lipid modification guidelines $^{2}$ state that either AST or ALT should be assessed prior to starting statins. The implications of this are that mildly elevated levels will be wrongly seen as a contraindication to the initiation of statins. This study, however, reinforces the concept that liver transaminases, and specifically an elevated AST/ALT ratio, should be seen as identifying those with a greater need for a statin because of increasing CVD risk rather than a contraindication because of the relatively rare occurrence of a statin-induced liver injury. ${ }^{31} 32$

\section{LIMITATIONS}

Imputation of three QRISK2 risk factors: BMI, Townsend deprivation score and ethnicity were required to preserve the integrity of the sample size. However, our analysis, using only Framingham risk factors which contained complete data, suggests that imputation did not significantly alter the results as there was similar discrimination between the Framingham and QRISK2 risk factor models. Ethnicity, in the original QRISK2 algorithm, comprises eight UK ethnic groups, but was limited to two groups in this analysis. More specific ethnic group recording in primary care records was particularly poor. While multiple imputation has limitations, in particular when data are not 'missing at random', the approach has been recommended for epidemiological studies and recognised to have to statistically validity in the development of primary care risk prediction tools. ${ }^{33}$

In addition, our results from modelling Framingham risk factors for predicting 10-year CVD risk showed that TC was not significantly associated with CVD while the original published Framingham risk model ${ }^{4}$ shows a significant association with CVD. This discrepancy can be explained by the fact that the Framingham cohort is 
derived from a true prospective cohort of individuals with an inclusion criterion of untreated, fasting cholesterol measured at baseline. Although we excluded patients on statins at baseline, it is impossible to distinguish between fasting and non-fasting levels from primary care computer records. However, we did find that the TC/HDL ratio was a significant and better predictor of CVD than TC alone, which supports the QRISK2 ${ }^{3}$ algorithm's use of the TC/HDL ratio instead of TC alone when developing risk algorithms from a primary care database.

Furthermore, other sources of AST include skeletal and cardiac muscle, with levels of AST increasing after acute MI. To account for this, we have excluded all patients at baseline with a history of CVD, including acute MI. Unless patients had undocumented MI in UK primary care records and secondary care linked records, which is highly unlikely given the significance of the event, this would not have likely been a confounding factor.

Finally, although the AST/ALT ratio is documented in a significant number of patients in this primary care database, there may be some ascertainment bias for measuring of these liver markers. Clinicians may have measured levels in patients who they suspect are at high risk of liver disease or as a marker for other confounding factors such as the decision to start and monitor statins and diabetes check-ups. However, the study cohort, comprising of a large sample of the UK general population, was not systematically different from the general population.

Acknowledgements The authors would like to thank Paula Dhiman for her methodological and statistical advice.

Contributors The study was conceived by SFW, NQ, JK, study designed by SFW and NQ. The primary author SFW wrote the first draft of the manuscript and the subsequent authors (JK, ING and NQ) contributed towards, revision, and preparation of the final manuscript. All four study authors have met authorship criteria set by International Committee of Medical Journal Editors (ICMJE).

Funding This work was supported by the Division of Primary, University of Nottingham and National Institute for Health Research School of Primary Care Research (NIHR-SPCR) and NIHR Nottingham Digestive diseases Biomedical Research Unit.

Competing interests None declared.

Ethics approval Clinical Practice Research Datalink Scientific Advisory Committee (CPRD-ISAC No. 14_080).

Provenance and peer review Not commissioned; externally peer reviewed.

Open Access This is an Open Access article distributed in accordance with the terms of the Creative Commons Attribution (CC BY 4.0) license, which permits others to distribute, remix, adapt and build upon this work, for commercial use, provided the original work is properly cited. See: http:// creativecommons.org/licenses/by/4.0/

\section{REFERENCES}

1. Townsend N, Wickramasinghe K, Bhatnagar P, et al. Coronary heart disease statistics 2012 edition. London: British Heart Foundation, 2012.
2. National Institute for Health and Care Excellence. Lipid modification: cardiovascular risk assessment and the modification of blood lipids for the primary and secondary prevention of cardiovascular disease. London: National Institute of Health and Care Excellence, 2014.

3. Hippisley-Cox J, Coupland C, Vinogradova Y, et al. Predicting cardiovascular risk in England and Wales: prospective derivation and validation of QRISK2. BMJ 2008;336:1475-82.

4. Wilson PW, D'Agostino RB, Levy D, et al. Prediction of coronary heart disease using risk factor categories. Circulation 1998;97:1837-47.

5. Ridker PM, Danielson E, Fonseca FA, et al., JUPITER Study Group. Rosuvastatin to prevent vascular events in men and women with elevated C-reactive protein. N Engl J Med 2008;359:2195-207.

6. Expert Panel on Detection Evaluation and Treatment of High Blood Cholesterol in Adults. Executive Summary of The Third Report of The National Cholesterol Education Program (NCEP) Expert Panel on Detection, Evaluation, And Treatment of High Blood Cholesterol In Adults (Adult Treatment Panel III). JAMA 2001;285:2486-97.

7. Nyblom $\mathrm{H}$, Berggren $\mathrm{U}$, Balldin J, et al. High AST/ALT ratio may indicate advanced alcoholic liver disease rather than heavy drinking. Alcohol Alcohol 2004;39:336-9.

8. Nyblom $\mathrm{H}$, Nordlinder $\mathrm{H}$, Olsson R. High aspartate to alanine aminotransferase ratio is an indicator of cirrhosis and poor outcome in patients with primary sclerosing cholangitis. Liver Int 2007;27:694-9.

9. Giannini E, Risso D, Botta F, et al. Validity and clinical utility of the aspartate aminotransferase-alanine aminotransferase ratio in assessing disease severity and prognosis in patients with hepatitis $C$ virus-related chronic liver disease. Arch Intern Med 2003;163:218-24.

10. Adams LA, Harmsen S, St Sauver JL, et al. Nonalcoholic fatty liver disease increases risk of death among patients with diabetes: a community-based cohort study. Am J Gastroenterol 2010;105:1567-73.

11. Adams LA, Lymp JF, St Sauver J, et al. The natural history of nonalcoholic fatty liver disease: a population-based cohort study. Gastroenterology 2005;129:113-21.

12. Ekstedt M, Franzen LE, Mathiesen UL, et al. Long-term follow-up of patients with NAFLD and elevated liver enzymes. Hepatology 2006;44:865-73.

13. Ong JP, Pitts A, Younossi ZM. Increased overall mortality and liver-related mortality in non-alcoholic fatty liver disease. J Hepatol 2008;49:608-12.

14. Soderberg C, Stal P, Askling J, et al. Decreased survival of subjects with elevated liver function tests during a 28-year follow-up. Hepatology 2010;51:595-602.

15. Angulo P, Hui JM, Marchesini G, et al. The NAFLD fibrosis score: a noninvasive system that identifies liver fibrosis in patients with NAFLD. Hepatology 2007;45:846-54.

16. McPherson S, Stewart SF, Henderson E, et al. Simple non-invasive fibrosis scoring systems can reliably exclude advanced fibrosis in patients with non-alcoholic fatty liver disease. Gut 2010;59:1265-9.

17. Angulo P, Bugianesi E, Bjornsson ES, et al. Simple noninvasive systems predict long-term outcomes of patients with nonalcoholic fatty liver disease. Gastroenterology 2013;145:782-9 e4.

18. Kim D, Kim WR, Kim HJ, et al. Association between noninvasive fibrosis markers and mortality among adults with nonalcoholic fatty liver disease in the United States. Hepatology 2013:57:1357-65.

19. Herrett E, Thomas SL, Schoonen WM, et al. Validation and validity of diagnoses in the General Practice Research Database: a systematic review. Br J Clin Pharmacol 2010;69:4-14.

20. British Cardiac Society, British Hypertension Society, Diabetes UK Heart UK, Primary Care Cardiovascular Society, The Stroke Association. JBS 2: Joint British Societies' guidelines on prevention of cardiovascular disease in clinical practice. Heart 2005;91(suppl 5):v1-52.

21. Newson R. Confidence intervals for rank statistics: somers' $D$ and extensions. Stata J 2006;6:309-34.

22. National Centre for Social Research and University College London. Department of epidemiology and public health. Health survey for England, 2012 [computer file]. Colchester, Essex: UK Data Archive [distributor], 2012.

23. Welch $\mathrm{C}$, Petersen I, Walters $\mathrm{K}$, et al. Two-stage method to remove population- and individual-level outliers from longitudinal data in a primary care database. Pharmacoepidemiol Drug Saf 2012;21:725-32.

24. Prati D, Taioli E, Zanella A, et al. Updated definitions of healthy ranges for serum alanine aminotransferase levels. Ann Intern Med 2002;137:1-10.

25. Lu H, Liu H, Hu F, et al. Independent Association between Nonalcoholic Fatty Liver Disease and Cardiovascular Disease: a Systematic Review and Meta-Analysis. Int $J$ Endocrinol 2013;2013:124958 
26. Ratib S, West J, Crooks CJ, et al. Diagnosis of liver cirrhosis in England, a cohort study, 1998-2009: a comparison with cancer. Am J Gastroenterol 2014;109:190-8.

27. Anstee QM, McPherson S, Day CP. How big a problem is non-alcoholic fatty liver disease? BMJ 2011:343.

28. JBS Board. Joint British Societies' consensus recommendations for the prevention of cardiovascular disease (JBS3). Heart 2014;100 (Suppl 2):ii1-67.

29. Helfand M, Buckley DI, Freeman M, et al. Emerging risk factors for coronary heart disease: a summary of systematic reviews conducted for the U.S. preventive services task force. Ann Intern Med 2009;151:496-507.

30. Stone NJ, Robinson J, Lichtenstein $\mathrm{AH}$, et al., American College of Cardiology/American Heart Association Task Force on Practice
Guidelines. 2013 ACC/AHA guideline on the treatment of blood cholesterol to reduce atherosclerotic cardiovascular risk in adults: a report of the American College of Cardiology/American Heart Association Task Force on Practice Guidelines. Circulation 2014;129 (25 Suppl 2):S1-45.

31. Chen GL, Hsiao FY, Dong YH, et al. Statins and the risk of liver injury: a population-based case-control study. Pharmacoepidemiol Drug Saf 2014;23:719-25.

32. Russo MW, Hoofnagle JH, Gu J, et al. Spectrum of statin hepatotoxicity: experience of the drug-induced liver injury network. Hepatology 2014;60:679-86.

33. Sterne JA, White IR, Carlin JB, et al. Multiple imputation for missing data in epidemiological and clinical research: potential and pitfalls. BMJ 2009;338:b2393. 